\title{
CORRIGENDUM
}

\section{Conditioning the genome identifies additional diabetes resistance loci in Type 1 diabetes-resistant NOR/Lt mice}

\author{
PC Reifsnyder, R Li, PA Silveira, G Churchill, DV Serreze and EH Leiter
}

Genes and Immunity (2006) 7, 184. doi:10.1038/sj.gene.6364292

Correction to: Genes and Immunity (2005) 6, 528-538. doi: $10.1038 /$ sj.gene. 6364241

Since the publication of the above article, the authors have identified an error in Figure 1 and its legend. Owing to a misleading genotyping, D4Mit310 was originally scored as NOD-derived indicating that the congenic possessed a shorter BKS-derived region than in the parental NOR. Figure 1c has been corrected to show that the congenic bears the full NOR-derived Chromo- some 4 congenic interval assessed in our study. This spans not only a region containing genes exerting the previously reported Idd11 and Idd9.1 locus effects (Refs. 11-13), but also that of the Idd9.2 locus. Markers are ordered according to Ensembl physical map location (in $\mathrm{Mb}$ ) rather than MGI linkage map position (in $\mathrm{cM}$ ).

The correct figure is shown below.

The authors would like to apologize for this mistake.
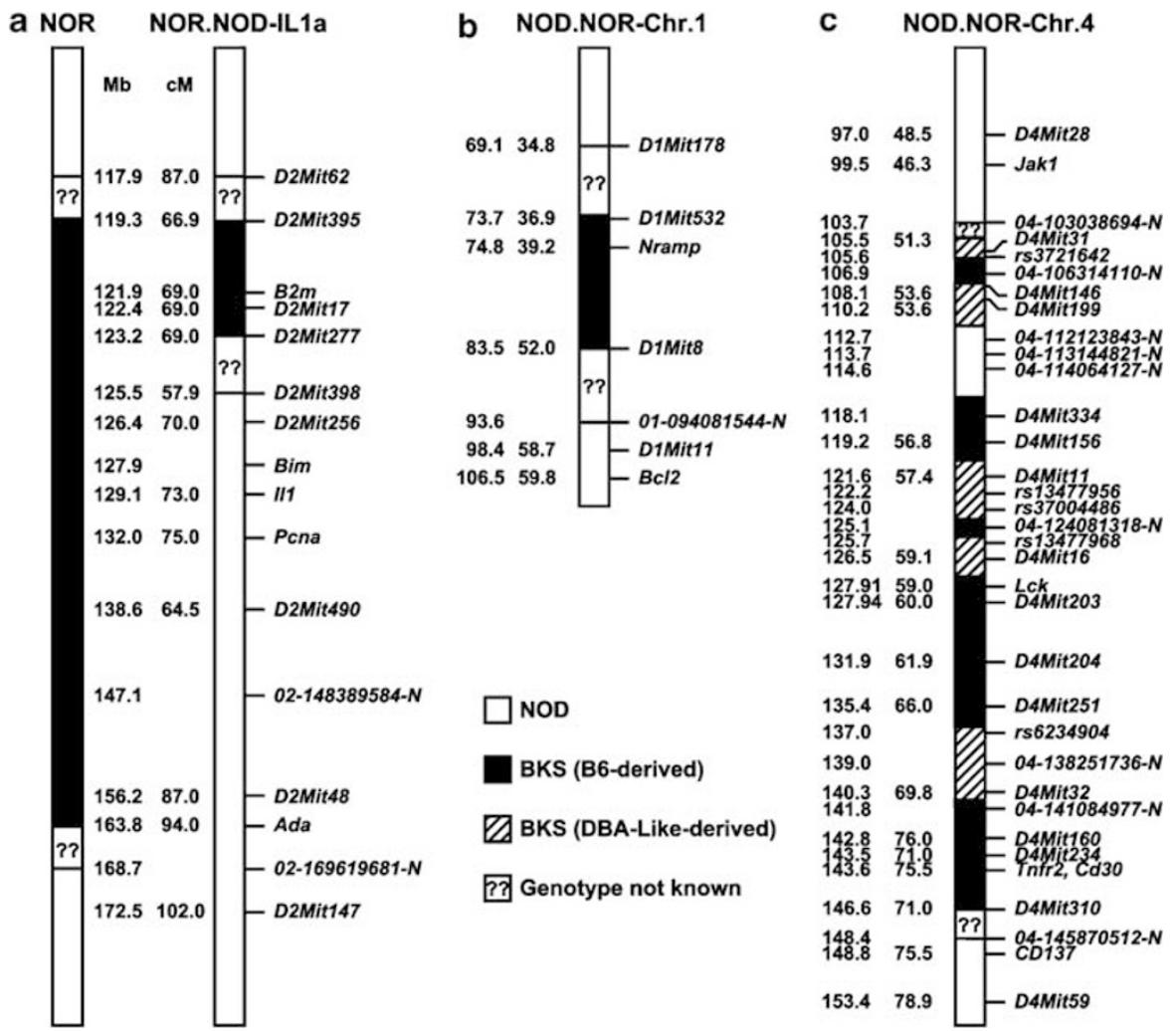

Figure 1 Schematic illustrations showing polymorphic markers defining congenic intervals. Genomic marker positions are shown in Mb (Ensembl, Build 33) and cM (MGI, The Jackson Laboratory), with B6 or DBA-derived regions from BKS indicated if known. All markers known to distinguish NOR from NOD in the relevant congenic regions are presented; those actually typed in congenic construction are described in Materials and methods. (a) Comparison of NOR Chr. 2 and the N21 NOR.NOD-Il1 congenic stock conditioned by replacement of NOR with NOD alleles below D2Mit277. (b) Length of NOR congenic interval in the NOD.NOR-Chr. 1 congenic stock. (c) Length of NOR congenic interval in the NOD.NOR-Chr. 4 congenic stock. 